\title{
QUESTIONS OF SIGNS IN POWER SERIES ${ }^{1}$
}

\author{
WOLFGANG B. JURKAT
}

This paper deals with the signs of the (real) coefficients of a power series $k(x)=\sum_{\nu=0}^{\infty} k_{\nu} x^{\nu}$, which is the quotient of the two power series $q(x)=\sum_{\nu=0}^{\infty} q_{\nu} x^{\nu}$ and $p(x)=\sum_{\nu=0}^{\infty} p_{\nu} x^{\nu}$. Questions of this type have been previously treated by Kaluza (1928) and Szegö (1926) in the special case of $q(x)=1$. Their significance for inclusion theorems for Nörlund means was shown by Hardy (1949), $\$ 4.5$.

By consideration of a general power series $q(x)$ instead of $q(x)=1$ it is possible to include the known results ${ }^{2}$ of this subject in a single theorem (\$1.1). Moreover the method of proof used allows us to establish a second type of theorem using simpler conditions (\$1.2). For this second type of theorem the introduction of a general $q(x)$ is essential for applications, because the results are still trivial for $q(x)=1$. There are two different principles to generalize. Thereby the second kind of theorem becomes also applicable in the case of $q(x)=1$ $(\S \S 2.1,2.2,2.4)$. The statements now obtained can even be used conversely for a second proof of the theorems first obtained (\$2.3).

The results of this paper are, as in the book of Hardy, fundamentally for application to inclusion of Nörlund means and related questions. These applications will be discussed in a following paper Some new inclusion theorems for Nörlund means. Also some concrete examples for the general theorems on distribution of signs will be found there.

1. Distribution of signs in $k(x)$. In the following we begin always with the formal product

$$
k(x) p(x)=q(x) \quad \text { or } \quad \sum k_{\nu} x^{\nu} \sum p_{\mu} x^{\mu}=\sum q_{n} x^{n},
$$

i.e. with the relation

$$
\sum_{\nu=0}^{n} k_{\nu} p_{n-\nu}=q_{n} \quad \text { for } n \geqq 0 .
$$

1.1. All the known results on the distribution of signs in $k(x)$ $=\sum k_{\nu} x^{\nu}$ are included in

Presented to the Society, November 28, 1953; received by the editors November 2, 1953 and, in revised form, March 8, 1954.

1 This research was supported by the United States Air Force under Contract No. 18(600)-691 monitored by the Office of Scientific Research.

${ }^{2}$ Another but similar question is that concerning the product of two power series instead of the quotient. Cf., for this, Davenport-Polya (1949). 
THEOREM 1. If $p_{n}$ and $q_{n}$ satisfy the conditions

$$
p_{n}>0 \quad(n \geqq 0), \quad \frac{p_{n+1}}{p_{n}} \nearrow \quad \text { for } 0 \leqq n \nearrow^{3}
$$

and

$$
\frac{q_{n}}{p_{n}} \nearrow \quad \text { for } 0 \leqq n \nearrow,
$$

then $k_{n} \geqq 0$ for $n \geqq 1$. (Hence it follows that either $\sum k_{n}=+\infty$ or $\sum k_{n}=\lim _{x \rightarrow 1-0} k(x)$, where both terms exist.)

To explain the contents of the theorem we consider first some specializations:

Because we placed no hypothesis on the signs of $q_{n}$, we may replace the terms $q_{n}, k_{n}$ in Theorem 1 by $-q_{n},-k_{n}$. In this way we obtain immediately

Theorem 2. If $p_{n}$ and $q_{n}$ satisfy (2) and

$$
\frac{q_{n}}{p_{n}} \searrow \quad \text { for } 0 \leqq n \nearrow^{3}
$$

then $k_{n} \leqq 0$ for $n \geqq 1$. (Hence it follows that either $\sum k_{n}=-\infty$ or $\sum k_{n}$ $=\lim _{x \rightarrow 1-0} k(x)$, where both terms exist.)

Theorem 2 clearly contains the following result ${ }^{4}$ of Kaluza (1928) and Szegö (1926).

Theorem 3. Let (2) hold and $q_{0}=1, q_{n}=0$ ( $\left.n \geqq 1\right)$ (the latter means $k(x)=1 / p(x)$ in the formal sense).

Then we have $k_{0}=1 / p_{0}>0, k_{n} \leqq 0(n \geqq 1)$. If moreover $p(x)=\sum p_{\nu} x^{\nu}$ is convergent for $|x|<1$, then it follows that $\sum k_{n}=\lim _{x \rightarrow 1-0} 1 / p(x) \geqq 0$, where both terms exist.

If in Theorem 1 we have always $q_{n}>0$ (or only $q_{0}>0$ ), then $k_{n} \geqq 0$ for $n \geqq 0$ holds, because of $k_{0}=q_{0} / p_{0}$. In this case the assertion of Theorem 1 can be taken from the proof of Hardy (1949) for his Theorem 23 (p. 69). By a short cut in the proof given there it is possible to deal with both cases $q_{n}<0$ and $q_{n}>0$ at the same time.

Proof of Theorem 1 . Let $n \geqq 1$ and if $n>1$ assume moreover $k_{1}, \cdots, k_{n-1} \geqq 0$. Then we have to show that $k_{n} \geqq 0$. By (1) we have

${ }^{3}$ The symbol “ $\nearrow$ " resp. “ $\searrow$ ” means increasing resp. decreasing (equality allowed).

${ }^{4}$ Cf. Hardy (1949), p. 68, Theorem 22. An example for such a distribution of signs is to be found in Knopp (1926), p. 331 . 


$$
\sum_{\nu=0}^{n-1} k_{\nu}\left(\frac{p_{n-\nu}}{p_{n}}-\frac{p_{n-1-\nu}}{p_{n-1}}\right)+k_{n} \frac{p_{0}}{p_{n}}=\frac{q_{n}}{p_{n}}-\frac{q_{n-1}}{p_{n-1}}
$$

and further by (2)

$$
\frac{p_{n-\nu}}{p_{n}}-\frac{p_{n-1-\nu}}{p_{n-1}} \begin{cases}=0 & \text { for } \quad \nu=0 \\ 0 & \text { for } \quad 1 \leqq \nu \leqq n-1\end{cases}
$$

Substituting in (5) gives by use of (3)

$$
k_{n} \frac{p_{0}}{p_{n}} \geqq \frac{q_{n}}{p_{n}}-\frac{q_{n-1}}{p_{n-1}} \geqq 0,
$$

1.2. An undesirable feature of the above theorem is the somewhat complicated condition (2). If $\sum p_{\nu} x^{\nu}$ is convergent for $|x|<1$ (as it will always be in later applications), then it follows from (2) that $p_{n}$ is decreasing. The next theorems will use this weaker condition.

ThEOREM 4. If $p_{n}$ and $q_{n}$ satisfy the conditions

$$
p_{0}>0, \quad p_{n} \searrow
$$$$
\text { for } 0 \leqq n \nearrow
$$

and

$$
\bar{\Delta} q_{n} \geqq \frac{q_{0}}{p_{0}} \bar{\Delta} p_{n} \quad \text { for } n \geqq 1,{ }^{5}
$$

then $k_{n} \geqq 0$ for $n \geqq 1$. (Hence it follows that either $\sum k_{n}=+\infty$ or $\sum k_{n}$ $=\lim _{x \rightarrow 1-0} k(x)$, where both terms exist.)

As in Theorem 1 there is no supposition on the sign of $q_{n}$, and therefore we may replace again $q_{n}, k_{n}$ by $-q_{n},-k_{n}$. This gives immediately

Theorem 5. Let (6) and

$$
\bar{\Delta} q_{n} \leqq \frac{q_{0}}{p_{0}} \bar{\Delta} p_{n} \quad \text { for } n \geqq 1 .
$$

Then we have $k_{n} \leqq 0$ for $n \geqq 1$. (Hence it follows that either $\sum k_{n}=-\infty$ or $\sum k_{n}=\lim _{x \rightarrow 1-0} k(x)$, where both terms exist.)

For the reciprocal power series $1 / p(x)$ we obtain directly from Theorems 4 and 5 only two rather trivial results.

Proof of Theorem 4 . Let $n \geqq 1$ and for $n>1$ assume moreover $k_{1}, \cdots, k_{n-1} \geqq 0$. Then we have to show that $k_{n} \geqq 0$. By (1) we have

${ }^{5}$ We use the abbreviations $\bar{\Delta} a_{\nu}=a_{\nu}-a_{\nu-1}(\nu \geqq 1), \bar{\Delta} a_{0}=a_{0}$. Then the condition (7) is satisfied of itself for $n=0$. 


$$
k_{0}\left(p_{n}-p_{n-1}\right)+\sum_{\nu=1}^{n-1} k_{\nu}\left(p_{n-\nu}-p_{n-1-\nu}\right)+k_{n} p_{0}=q_{n}-q_{n-1}
$$

and further

$$
k_{0}=\frac{q_{0}}{p_{0}}, \quad p_{n-\nu}-p_{n-1-\nu} \leqq 0 \quad \text { for } 1 \leqq \nu \leqq n-1 .
$$

Substituting in (9) and applying (7) we obtain

$$
k_{n} p_{0} \geqq \bar{\Delta} q_{n}-\frac{q_{0}}{p_{0}} \bar{\Delta} p_{n} \geqq 0,
$$

1.3. We now compare briefly the conditions of the Theorems 1 and 4. As already mentioned, (6) is in general weaker than (2). Of the other conditions-as a compensation so to speak-(7) is in general stronger than (3). More precisely we have

Theorem 6. If $0<p_{n} \searrow$ for $0 \leqq n \nearrow$, then (3) follows from (7).

If on the contrary $0<p_{n} \nearrow$ for $0 \leqq n \nearrow$, then conversely (7) follows from (3).

Proof. First we state at once the equivalence of the inequalities

$$
\frac{q_{n}}{p_{n}} \geqq \frac{q_{n-1}}{p_{n-1}} \quad \text { and } \quad \bar{\Delta} q_{n} \geqq \frac{q_{n-1}}{p_{n-1}} \bar{\Delta} p_{n}
$$

for $n \geqq 1$. Further by (3) or by (7) we have

$$
\frac{q_{n-1}}{p_{n-1}} \geqq \frac{q_{0}}{p_{0}}
$$

for $n \geqq 1$.

Both together give the assertion with regard to the sign of $\bar{\Delta} p_{n}$.

2. Some extensions.

2.1. We have previously defined $k(x)$ by the formal quotient $q(x) / p(x)$. But in applying the Theorems 1 and 4 we can use as well the representation

$$
k(x)=\frac{q(x) r(x)}{p(x) r(x)}=\frac{\bar{q}(x)}{\bar{p}(x)} \text { with } r(x)=\sum r_{\nu} x^{\nu},
$$

and this gives

THEOREM 7. If there is a sequence $r_{n}$ such that the sequences

$$
\bar{q}_{n}=\sum_{\nu=0}^{n} q_{\nu} r_{n-\nu}, \quad \bar{p}_{n}=\sum p_{\nu} r_{n-\nu},
$$


instead of $q_{n}, p_{n}$, satisfy the conditions (2), (3) or (6), (7), then $k_{n} \geqq 0$ for $n \geqq 1$.

The proof follows from the relation

$$
\sum_{\nu=0}^{n} k_{\nu} \bar{p}_{n-\nu}=\bar{q}_{n} \quad \text { for } n \geqq 0,
$$

which we have by (1) and (11).

Corresponding to this we can also have extensions of Theorems 2,3 , and 5 .

We obtain simple and at the same time general conditions by setting $r(x)=(1-x)^{\alpha}$, i.e.

$$
r_{n}=(-1)^{n}\left(\begin{array}{l}
\alpha \\
n
\end{array}\right)=\left(\begin{array}{c}
n-\alpha-1 \\
n
\end{array}\right), \quad \alpha \text { any real number. }
$$

In this case we shall write

$$
\bar{q}_{n}=\bar{\Delta}^{\alpha} q_{n}, \quad p_{n}=\bar{\Delta}^{\alpha} p_{n} .
$$

For example we formulate the part of Theorem 7, which corresponds to Theorem 4.

THEOREM 8. If there is a real number $\alpha$ such that

$$
\begin{array}{cr}
p_{0}>0, \quad \bar{\Delta}^{\alpha} p_{n} \searrow & \text { for } 0 \leqq n \nearrow, \\
\bar{\Delta}^{\alpha+1} q_{n} \geqq \frac{q_{0}}{p_{0}} \bar{\Delta}^{\alpha+1} p_{n} & \text { for } n \geqq 1
\end{array}
$$

are satisfied, then $k_{n} \geqq 0$ for $n \geqq 1$.

2.2. In some cases it is not possible to have results directly concerning the distribution of signs in $k(x)$, but only in $k(x) r(x)$. By application of the Theorems 1 and 4 we find

THEOREM 9. If there is a sequence $r_{n}$ such that the sequences

$$
\bar{q}_{n}=\sum_{\nu=0}^{n} q_{\nu} r_{n-\nu} \text { and } p_{n},
$$

instead of $q_{n}, p_{n}$, satisfy the conditions (2), (3) or (6), (7), then $\vec{k}_{n}$ $=\sum_{\nu=0}^{n} k_{\nu} r_{n \rightarrow \nu} \geqq 0$ for $n \geqq 1$.

The proof follows at once from the relation

$$
\sum_{\nu=0}^{n} k_{\nu} p_{n-\nu}=\bar{q}_{n} \quad \text { for } n \geqq 0 .
$$


The situation is very similar to that of Theorem 7, and the examples shown there may be used in this case too. Thus instead of Theorem 8 we have

THEOREM 10. If there is a real number $\alpha$ such that condition (6),

$$
p_{0}>0, \quad p_{n} \searrow \quad \text { for } 0 \leqq n \nearrow,
$$

and

$$
\bar{\Delta}^{\alpha+1} q_{n} \geqq \frac{q_{0}}{p_{0}} \bar{\Delta} p_{n} \quad \text { for } n \geqq 1
$$

are satisfied, then $\bar{\Delta}^{\alpha} k_{n} \geqq 0$ for $n \geqq 1$.

COROLlaRY. If instead of (19) with $\alpha=-1$ we require the stronger condition

$$
q_{n} \geqq 0 \quad \text { for } n \geqq 0,
$$

then $\sum_{\nu=0}^{n} k_{\nu} \geqq 0$ for $n \geqq 0$.

For the proof put $r(x)=(1-x)^{\alpha}$ in Theorem 9 and use the conditions (6), (7).

The corollary refers to the especially simple case $\alpha=-1$. The condition (20) is satisfied e.g. for $q(x)=1$, i.e. in the case of the reciprocal power series $k(x)=1 / p(x)$. This example shows what may be deduced from the condition (6) alone.

2.3. The last remark enables us to add a second proof of Theorem 4: First let formally $1 / p(x)=\sum_{\nu=0}^{\infty} \gamma_{\nu} x^{\nu}$, i.e.

$$
\sum_{\nu=0}^{n} p_{\nu} \gamma_{n-\nu}=\left\{\begin{array}{lll}
1 & \text { for } & n=0 \\
0 & \text { for } & n \geqq 1
\end{array}\right.
$$

Putting $\Gamma_{n}=\sum_{\nu=0}^{n} \gamma_{\nu}$ we have then for $n \geqq 1$

$$
p_{0} \Gamma_{n}+\sum_{\nu=1}^{n} \bar{\Delta} p_{\nu} \Gamma_{n \rightarrow \nu}=0,
$$

which by induction gives $\Gamma_{n} \geqq 0$ for $n \geqq 0$ because of (6). On the other hand we have formally $k(x)=q(x)(1 / p(x))$, hence for $n \geqq 1$

$$
k_{n}=\sum_{\nu=0}^{n} q_{\nu} \gamma_{n-\nu}=q_{0} \Gamma_{n}+\sum_{\nu=1}^{n} \bar{\Delta} q_{\nu} \Gamma_{n-\nu} .
$$

Then from (7) and (22) follows

$$
k_{n} \geqq \frac{q_{0}}{p_{0}} \cdot p_{0} \Gamma_{n}+\sum_{\nu=1}^{n} \frac{q_{0}}{p_{0}} \cdot \bar{\Delta} p_{\nu} \Gamma_{n-\nu}=0, \quad \text { q.e.d. }
$$


This method of proof is related to the method of Hardy's proof ${ }^{6}$ for the special case of Theorem 1 previously mentioned.

2.4. As we have just now seen the considerations of signs in the reciprocal series $1 / p(x)$ are fundamental for the theorems stated. Therefore it is interesting that we may get further theorems by combination of the methods of Theorems 7 and 9, which are applicable to the reciprocal series. For simplicity we restrict ourselves to a combination of the Theorems 8 and 10, which corresponds essentially to Theorem 4. There is of course a similar theorem resulting from Theorem 1.

Theorem 11. If there are two real numbers $\alpha, \beta$ such that

$$
p_{0}>0, \quad \bar{\Delta}^{\alpha} p_{n} \searrow \quad \text { for } 0 \leqq n \nearrow
$$

and

$$
\bar{\Delta}^{\alpha+\beta+1} q_{n} \geqq \frac{q_{0}}{p_{0}} \bar{\Delta}^{\alpha+1} p_{n} \quad \text { for } n \geqq 1
$$

are satisfied, then $\bar{\Delta}^{\beta} k_{n} \geqq 0$ for $n \geqq 1$.

COROLlaRY. If instead of (25) with $\beta=-\alpha-1$ we require the stronger condition

$$
q_{n} \geqq 0 \quad \text { for } n \geqq 0,
$$

then $\bar{\Delta}^{-\alpha-1} k_{n} \geqq 0$ for $n \geqq 0$.

The proof is trivial.

\section{REFERENCES}

H. Davenport and G. Pólya

(1949) On the product of two power series, Canadian Journal of Mathematics vol. 1, pp. 1-5.

G. H. HARDY

$$
1 \text {, pp. 1-5. }
$$

(1949) Divergent series, Oxford.

TH. KALUZA

(1928) Über die Koeffizienten reziproker Potenzreihen, Math. Zeit. vol. 28, pp. 161170.

K. KNOPP

(1926) Über Polynomentwicklungen im Mittag-Leflerschen Stern durch Anwendung der Eulerschen Reihen-transformation, Acta Math. vol. 47, pp. 313-335.

G. SzEGö

(1926) Bemerkungen zu einer Arbeit von Herrn Fejér über die Legendreschen Polynome, Math. Zeit. vol. 25, pp. 172-187.

UNiversity OF CincinNati

' Cf. Hardy (1949), p. 69, Theorem 23. 